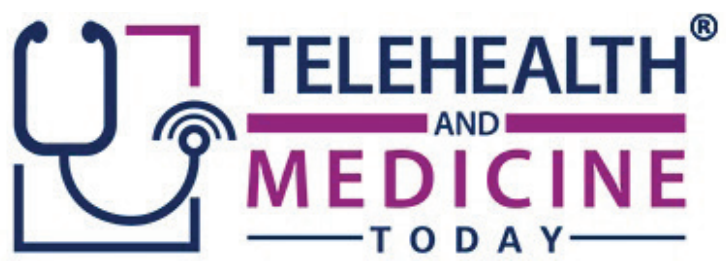

\title{
Telemental Health Care: A Review of Efficacy and Interventions
}

\section{Calvin T. Schaffer, BS ${ }^{1}$, Preeti Nakrani, MPH ${ }^{2,3}$, Paul A. Pirraglia, MD MPH ${ }^{1,2,3}$}

Affiliations: ${ }^{1}$ University of Massachusetts Medical School-Baystate; ${ }^{2}$ BeHealthy Partnership; ${ }^{3}$ Baystate Health

Corresponding Author: Calvin T. Schaffer, BS; https://orcid.org/0000-0002-4958-2967; calvin.schaffer@ umassmed.edu

Keywords: Telemedicine, Mental Health, Health Services Accessibility, Emergency Service, Hospital

Section: Use Cases

Objective: Mental illness continues to rise in the United States, burdening a healthcare system set to dive further into a shortage of mental health practitioners. The effects of this are already being felt in many rural areas, which are all too frequently understaffed to address the mental health concerns of their populations. To further compound this growing crisis, COVID-19 has led to a reduction in access to in-person care. Furthermore, COVID-19 has led to reduced access to in-person care. As a result, Telehealth has become more essential. Knowledge of the strategies and barriers to implement a successful Telehealth program is necessary to deliver a sustainable, accessible, and quality care.

Design: In this review, we analyze published research on the efficacy of Telehealth for mental health, discuss how these services have been implemented, and explore how to address barriers to quality care delivery via Telehealth.
Results: Telehealth, when the appropriate resources and supports are considered, is effective in a wide range of patient populations and care locations. Multiple modalities, including via video, apps, and telephone were shown to be efficacious. Interventions have been shown to increase the accessibility to care without compromising quality of care.

Conclusions: Telehealth constitutes a wellresearched, efficacious tool to address the issues in access to care. Telemental health programs should address the barriers to implementation, including training, access to technology, reimbursement and regulations, and adequate program oversight. Telehealth interventions should be strongly considered in areas facing shortages of mental health practitioners and long wait times for patients with mental health disorders, to reduce the burden of mental illness on healthcare. 


\section{INTRODUCTION}

In 2018, one in five US adults, or 47.6 million people, experienced mental illness and less than half of those people received treatment. ${ }^{1}$ The toll on the US healthcare system is immense, accounting for one out of every eight emergency department (ED) visits. ${ }^{1}$ Unfortunately, these patients remain vulnerable to relapses and costly readmissions following discharge. Follow-up care by trained mental and behavioral health professionals is therefore important to maintain the well-being of patients with mental illness diagnoses. ${ }^{2}$ For those in rural areas, the ability to connect to a mental health professional is extremely limited, resulting in an increased burden on rural primary care physicians and increased utilization of the internet by patients for medical advice. ${ }^{3}$ Additionally, many patients are averse to going into the hospital, a consideration that holds even more dramatic effect given the impact of COVID-19. The April 2020 poll by the American College of Emergency Physicians demonstrated that $29 \%$ of Americans were avoiding or delaying medical care for the fear of COVID-19. ${ }^{4}$ Another poll by the Kaiser Family Foundation found that $48 \%$ of adults indicated that they or someone in their household has rescheduled or passed up medical appointments over the course of 3 months as a result of COVID-19. ${ }^{5}$ Beyond this, COVID-19 has resulted in a considerable mental health burden and has made in-person appointments impossible in many locations. For those living in remote areas or who lack adequate time, money, and transportation services necessary to receive care, Telemental health provides a valuable alternative. ${ }^{3}$

\section{METHODS}

We performed a narrative review of the literature on Telemental health to date. We used PubMed and Google Scholar with the search terms Telemental health, Telehealth and mental health, and variations that included searches for Telehealth implementation, interventions, and barriers for use in mental health care. Given the breadth of terminology used to describe these modalities of virtual care, searches were expanded to include published materials on Telehealth, Telemental health, Telemedicine, Telepsychiatry, Telebehavioral health, Telemonitoring, telephone, digital health, virtual care, and remote care. These terms, while similar, are not synonyms. The variation in terms provided here stems from differences in the specific studies performed by the authors cited. All the available published articles focusing on the use of technology in the delivery of mental and behavioral health care were obtained and considered. Articles with a pay-wall or language barrier, were excluded. All articles were listed, sorted by relevance in the judgement of the authors, and categorized by topic. Findings, statistics, and implications were analyzed, collated, and organized, respectively. Here, we summarize our findings to provide a comprehensive view of Telehealth and its uses in mental health care.

\section{RESULTS}

\section{The Efficacy of Telehealth as a Care Modality}

Telehealth is a method of remote delivery of medical services, which provides a way for a provider to diagnose and treat individuals without a face-to-face consultation. ${ }^{6}$ Telehealth has been shown to be effective and satisfactory for a variety of patients, with patients in some cases providing even more information than they would in an in-person setting. ${ }^{3}$ Among lowincome patients in a rural health professional shortage area, distance to access points did not predict differences in service utilization, however, these patients did have to travel farther for these resources. The study supports the need to consider geographic, socioeconomic, transportation, and health-related barriers which 
different patient groups face and suggests the "hub and spoke" Telehealth model may be beneficial for rural and underserved communities. ${ }^{7}$ Still, many studies affirm that Telehealth is not a substitute for all in-person care and that clinicians must evaluate for which conditions, patients, and appointments Telehealth is most appropriate. Despite concerns, Telehealth has been shown to have significant positive statistical and clinical impacts and users of Telehealth are able to quickly adapt, provide necessary information as they would in person, and beneficially connect with their provider. ${ }^{3}$ Among providers, a study by Mace et al. found that the majority of respondents believed Telehealth was important to the success of their organization and a valuable tool for improving access and quality of care. ${ }^{8}$

\section{The Role of Telehealth in Mental Health Care}

Telehealth has been used significantly by psychiatrists and mental health counselors, who largely use direct video conference modality. A review by the Agency for Healthcare Research and Quality identified evidence that supports the effectiveness of Telehealth for psychotherapy. ${ }^{8}$ This includes videoconferencing, online forums, smartphone apps, text-messaging, and e-mails as useful communication methods for the delivery of mental health care both prior to and during COVID-19. ${ }^{9}$ Past reviews of Telemental healthcare indicate that it is cost effective, efficient, adaptable, and useful at providing promising outcomes for mental health patients. ${ }^{3,10}$ This holds true especially for under-developed communities where there is a need to improve access and deliver Telemental health care along with the usual conventional care. ${ }^{10}$ Indeed, among the earliest uses for Telemental health were to address limited access among rural patients who do not have the logistical or financial ability to attend in-person appointments. It is not surprising that among this patient population, participants would choose a Telemental health program over all other options, and especially, over not receiving treatment at all. ${ }^{11}$

The uses of Telemental health are vast and it has been shown to be effective for diagnosis and assessment for a wide-range of disorders, populations, and settings. Such efficacy has been shown for a variety of disorders including social anxiety disorder, posttraumatic stress disorder (PTSD), and Panic Disorder. ${ }^{12-14}$ A study that conducted technology-assisted cognitivebehavioral therapy (CBT) for symptoms including depression, pain, and fatigue in patients with chronic illnesses in end-stage renal disease required few resources and resulted in high patient adherence, satisfaction, and variable improvements. The intervention was feasible and well accepted, with seven of the eight participants completing all the eight prescribed sessions and agreeing that they were comfortable in the sessions and found them to be helpful. ${ }^{15}$ A similar study focusing on the impact of trauma-focused CBT via Telehealth found that this model resulted in statistically significant clinical improvements and high satisfaction ratings from providers and patients comparable to office-based settings. ${ }^{16}$ Carpenter et al. demonstrated that delivering family-based cognitive therapy via videoconferencing directly to the patient's home was shown to have high treatment retention, high client satisfaction, strong therapeutic alliance, and low barriers to participation for treatment of child anxiety. ${ }^{17}$ Beyond CBT, a randomized controlled study found a comprehensive online depression care management program resulted in improved outcomes including lowered depression and better overall mental health, increased satisfaction with mental healthcare and coping skills, and increased confidence in handling depression. ${ }^{18}$ Although Telehealth post-exposure treatment has been safely and successfully used to treat symptoms 
of PTSD and depression in veterans, Tuerk et al. found that this intervention led to an increase in non-completion rates as compared with in-clinic treatment, though it was also associated with large reductions in symptoms. ${ }^{19}$

Generally, attitudes and perceptions toward Telehealth have been generally receptive for both urban and rural primary care patients. Although more than half of rural and urban patients do not view Telepsychiatry to be as helpful as face-to-face interventions, the vast majority still believe that Telepsychiatry services are helpful and nearly half would use the services if it would save them a two-hour drive. Additionally, the majority of patients have some comfort with using the technology and access to a telephone, computer, and Internet access at home. ${ }^{20} \mathrm{~A}$ study by Gibson et al. regarding the satisfaction of dementia patients and their caregivers found that services within the home setting were less distressing, confusing, and onerous and more empowering than being transported to a clinic. ${ }^{21}$ As a whole, published scientific literature shows strong and consistent evidence of feasibility, cost effectiveness, acceptance, and improved symptomology and quality of life for the use of Telemental health for a wide range of demographic and diagnostic groups. ${ }^{22}$ Telemental health improves access to care, especially in areas with limited mental health resources. Using a collaborative framework, clinical and non-professional providers can effectively provide behavioral therapies with increasing cost effectiveness over time and with increased integration and use. ${ }^{23}$ Thus, when correctly integrated and maintained, Telehealth appears to be a viable and realistic approach in treating mental health illness and positively impacting the quality, timeliness, and availability of services in almost any mental health care system. ${ }^{3}$
The Substance Abuse and Mental Health Services Administration (SAMHSA) identified examples of Telehealth services that include online substance use questionnaires, CBT through video conferencing, text message reminders to take medications as directed, group chats for relapse prevention, webinars for clients and providers, and interactive video for consultation. A SAMHSA publication reported that telephones have been shown to improve treatment and medication compliance when used in monitoring recovery from mental illness and substance use disorder. Telephone interventions also resulted in reduced symptoms of anxiety and depression and were effective at motivating patients to enter treatment. ${ }^{8,23}$ In a 3-month randomized controlled trial, the use of a smartphone-delivered intervention led to an increase in starting treatment from 58\% to $90 \%$ and continued engagement from $40 \%$ to $56 \%$ as compared with a clinic-based group intervention among those with general psychopathology and depression, with additional findings of improved recovery and quality of life after 6 months for the intervention group. ${ }^{24}$ Indeed, in addition to web-based therapy, telephone services can be used in conjunction with computer-based assessments and support. In one study, patient feedback to a telephone intervention was positive overall and there were high rates of consent and retention with patients enjoying the accessibility, convenience, and privacy of the modality. The positive results indicate a role for telephone as a mode of health care delivery and the need for further research into the optimal integration of telephone services in patient care. ${ }^{25}$

Telemental health can improve access to follow-up appointments, though considerations must be made to ensure the success of these interventions. A mental health facility found that although access to care was improved and there was overall satisfaction and continued willingness 
to use Telehealth, they found issues with compliance due to limited appointment times and options. ${ }^{6}$ In terms of HEDIS metrics for follow-up after hospitalization for mental illness within seven days post-discharge for 2018, Commercial HMO has a rate of $45.6 \%$, Commercial PPO is at $43 \%$, Medicaid HMO is at $35.8 \%$, Medicare $\mathrm{HMO}$ is at $27.7 \%$, and Medicare PPO is at $27.3 \%$. $^{2}$ This suggests that vulnerable populations including the elderly and poor are receiving follow-up care far less than other patient groups, a reality that can be lessened through the use of Telemental health when applied properly. Managed care company, Aetna, proposes maintaining appointment availability for recent hospital discharges, explaining the importance of follow-up, reaching out to patients who do not keep initial follow-up appointments and quickly and appropriately rescheduling them, and using Telehealth to connect patients with a qualified mental health practitioner. In addition to these, Aetna provides additional strategies for follow-up after ED discharge with mental illness, recommending that the follow-up appointment is scheduled before the patient leaves and that Telehealth can be used by any type of practitioner. ${ }^{26}$ Although Telemental health has been shown to be effective at reducing unnecessary travel to urban centers and satisfactory to patients and families for safe emergency assessment and follow-up, the barriers that lead to health inequities must be identified and addressed for this healthcare modality. ${ }^{27}$

In remote and rural settings, a community-based Telepsychiatry program can be useful in bringing psychiatrists for consultations. One such study focused on the use of such a program in the care of older adults with a broad range of psychiatric disorders as a supplemental means of care to an overstretched visiting service. This program was well accepted among patients and providers, however, satisfaction among patients admitted in hospital was lower than for outpatients and illness and sensory disabilities results in decreased satisfaction. The use of mobile Telepsychiatry within nursing homes and linked to the secure video-conferencing system could provide a facilitating environment for geriatric patients to receive Telemental health care. ${ }^{28}$ Another study that provided veterans with mental health conditions with video-enabled tablets to conduct Telemental health services reported a 1.94 increase in the number of patient-provider encounters, a 1.05 increase in medication management visits, an $18.5 \%$ increase of receiving mental health care related to continuity of care, and a $20 \%$ decrease in their missed opportunity rate. ${ }^{29}$ By increasing access and continuity of care while decreasing missed opportunities for care, providing patients with the means to use Telemental health is another possible intervention. ${ }^{29}$ Even the use of mobile apps and SMS text messaging as mental health interventions has been found to be feasible, usable, and efficacious. These interventions have had promising results for self-care and to encourage a healthier lifestyle, promote adherence, improve compliance, and reduce healthcare visits. In some cases, apps were shown to have a more significant effect on medication adherence and psychoeducational information was more easily understood when read in SMS form. The effectiveness of such interventions has been demonstrated both empirically and by the perceptions of the patients. Still, as with other modes of Telemental health, access and ability to use virtual features is constrained by socioeconomic factors including internet access and data usage. ${ }^{30}$

Many studies have explored the use of Telemental health services directly in the ED. ${ }^{31}$ This stems from concerns over the costs of mental health ED visits, their prominence 
among those entering the ED, and limited resources in many ED facilities and under resourced communities to cope with the increasing demand for mental health services. Mental health accounts for over $10 \%$ of ED visits, and that number is expected to increase to $23 \%$ in the next decade. ${ }^{32,33}$ Telehealth consultation for behavioral health has been associated with decreased wait time and an increase in routine discharge for Telebehavioral patients with a tailored safety plan, however, it has also been shown to lead to longer ED length of stay (LOS) and higher relative costs for substance abuse cases. ${ }^{31}$ This has prompted a discussion on the use of Telemental health to better understand individual patient needs and lead to more efficient care for different behavioral health conditions as well as its use in rural, remote, and under-resourced hospitals in need of psychiatric professional support. ${ }^{31}$ In two studies, Mohr et al. found that rural hospital EDs that implemented a telemedicine network in the Midwest US were more efficient in timeliness of care and LOS for physical health conditions, with a 6- min decrease in door-toprovider time and a nearly 15 -min decrease when seen by a Telemedicine provider, although total ED LOS varied by patient type. ${ }^{34,35}$ Rural patients have particular difficulty in accessing mental health services in the ED. Using videoconferencing may mitigate this by connecting these patients with mental health professionals. At one clinic in Southwestern Ontario, ED staff perceived video conferencing to be useful in improving patient experience, benefiting crisis response teams, and at expediting the direct assessment of patients with mental health emergencies by mental health specialists. There were concerns however that these services strained ED resources and that lack of use and difficulties in assessing when these resources should be used were barriers to successful implementation. ${ }^{36}$
Despite this support, some Telemonitoring interventions have not resulted in fewer hospitalizations or ED visits or better case management outcomes despite increased costs for support and equipment, including a study by Takahashi et al., which found no significant difference between Telemonitoring and usual care for older patients and actually noted an increase in events and mortality of unknown cause. ${ }^{37}$ ED providers have expressed concerns about lacking mental health training, resources, and access to mental health professionals to address what has been a growing need for mental health services. Coordinating virtual care through a centralized Telemedicine activation, dispatch, and admission and transfer center could help address these resource and access concerns. ${ }^{38}$ A behavioral health program that implemented a virtual navigation program that consists of a video contact with a patient while in the ED and then a follow-up by telephone 24-72 h after discharge showed a reduction in admissions (55.1-63.1\%) and a decrease in follow-up self-harm diagnoses (36.8-45.5\% as compared with usual care. ${ }^{39}$ Using a central hub and triage system, a number of regional sites can be provided by a team of psychiatry consultants and social workers, with services distributed efficiently and effectively where needed. While this system has been shown to be successful, it relies on the standardization of social work triage, protocol for patient management, and protocol for patient safety and security in the ED and due to its complexities, it should be implemented in phases to regional EDs. ${ }^{38}$ These types of interventions can then even be scaled up to the statewide level. In South Carolina, a Telepsychiatry program resulted in an increase in the likelihood of outpatient follow-up from 16\% to $46 \%$ after 30 days and $20 \%$ to $54 \%$ after 90 days, decreased admission to the inpatient setting at their ED visit from $22 \%$ to $11 \%$, a 0.86 reduction in LOS at that visit, and a $\$ 2,336$ 
reduction of overall 30 day inpatient costs as compared with the control group. ${ }^{40}$

Barriers to the implementation of Telemental health include implementation and maintenance costs, reimbursement concerns, the need for organizational structure and integration into workflow, workforce shortages, especially for behavioral health professionals, and educational and training needs. This illustrates the importance of an organized roll-out of Telemental health interventions. To ensure this occurs, necessary infrastructure must be developed and supported, adequate staff resources and training through initiatives to enhance the understanding of Telehealth rules, regulations, and best practices should be offered, and patient resources should be developed and widely disseminated. ${ }^{8}$ The Telehealth Resource Centers provides assistance, educational materials, and information for providers, administrators, and organizations. ${ }^{41}$ Another potentially useful resource is Project Extension for Community Healthcare Outcomes (ECHO), a collaborative model that is used to train healthcare providers and staff using virtual meetings, webinars, information sharing, and remote consulting. ECHO focuses on complex conditions management including behavioral health disorders and treatment access challenges, including those seen in rural and underserved areas. ${ }^{42}$ For mobile applications, the Addiction Technology Transfer Center Network has been reported to be helpful in better understanding the technology. 8,43

Patient challenges to be considered include lack of technology and resources, a lack of transportation (to begin a provider-patient relationship), and a lack of health insurance coverage. Based on this, allowing healthcare services to begin without a face-to-face interaction can improve access to care by reducing transportation barriers. Making Telehealth services compatible with mobile phones is another consideration to help address technology inequities. ${ }^{8}$ Language barriers, a major obstacle in health care delivery, need to be overcome in Telemental health interventions as they do in an in-person setting. Adoption of multi-language apps, e-tools, educational materials, and mental health information on a Telemental health platform can help to increase accessibility and availability of care among multicultural communities. ${ }^{44}$ Culture may also play a role in the likelihood of patient adoption and should be considered when developing digital health technology for use in a population. ${ }^{45}$ With these challenges in mind, Zhou et al. note that a focused public education campaign to promote these services may be useful in spreading awareness and increasing accessibility, adoption, and usability of the technology. ${ }^{9}$

Use of mobile phones or computers for video conferencing has strengthened mental health management and follow-up. However, the field of Telemental healthcare and potential interventions is expansive and not limited to phone calls or video chats. Imaging capabilities, psychotherapy, clinician consultations, home care, automated evaluations, behavioral analysis, and virtual information exchange further enhance Telemental health modalities. ${ }^{10}$ Additional considerations must be made, for example, training mental health professionals and other providers, handling technical difficulties, protocol on "no-shows," record keeping, figuring out how to best provide questionnaires and assessments, developing secure and easy methods to obtain signatures for confidentiality, dissemination of patient information when necessary, ensuring privacy, and reimbursement must be addressed at the onset of implementation of Telemental health services. ${ }^{3}$ It is also imperative to provide therapists and providers 
with appropriate training and to address privacy and confidentiality concerns when using Telemental health interventions. ${ }^{11}$ As a result of these concerns and potential limitations, it is necessary to provide patients with full disclosure of the benefits and barriers of Telemental health and making an informed decision together about whether or not it would improve their care and should be used. ${ }^{3}$ Langarizadeh et al. recommend robust feedback from both patients and providers to address concerns like these and evaluate any Telemental health program to improve its use in the context of specific healthcare systems. ${ }^{10}$

Additional concerns tend to stem from difficulties in the implementation of Telehealth technology on both the patient and provider sides, including internet access, ineffective implementation of a new technology into the healthcare process, unclear quality controls and standards, regular evaluations and upgrades to the system and its modalities, and the constantly changing nature of reimbursement for new types of medical care modalities such as Telehealth by phone and video. ${ }^{10}$ On a system level, although Telehealth has demonstrated a history of cost effectiveness for nonphysical care, such as clinical interviews by psychiatrists as demonstrated in Shore et al., coverage for this care may be uncertain. ${ }^{46}$ COVID-19 has led to increased reimbursement for these services across the nation, however, there is hesitancy among providers over the longevity of these changes and, as such, reimbursement concerns must remain a consideration for new Telehealth programs. ${ }^{47}$ On an individual level, clinician reluctance to adopt new and potentially confusing technology, concerns about marginalizing patient populations that might have difficulty in using the new tools, and those who may not have access to the services altogether due to lacking resources present further barriers that any health system adopting Telemental health services must overcome. In some instances, in-person appointments may be the only feasible option, presenting evidence that Telemental health services constitute an effective supplementation to a patient's care but may not be a complete replacement for some traditionally in-person services. Knowledge of the capabilities of clinics to provide access to Telemental health resources, fostering clinician and patient understanding about the pros and cons of such technology and when it should be used, and seamless integration into normal process workflows are important considerations when implementing a Telemental health program or service. ${ }^{10}$

It is important to note that some groups of patients may initially be less open and willing to use some forms of Telemental health services in place of in-patient visits. A study of veterans' attitudes about the potential use of Telepsychiatry for mental health treatment in routine clinical settings found that $25.7 \%$ of patients were "not at all comfortable" and $13.4 \%$ were "extremely comfortable" using Telepsychiatry from home, and just $32.8 \%$ of participants indicated a clear preference for Telepsychiatry compared with in-person mental health visits, despite no differences in Telepsychiatry preferences by symptom severity, demographic variables, rural versus urban location, or endorsed barriers to care. ${ }^{48}$ Due to COVID-19, however, increased usage of this modality may lead to more user comfort and understanding of the technology. ${ }^{49}$ For patients with sensory disabilities and other illness-related deficits that might make Telemental health difficult, portable devices, hearing or visual aids, background noise cancellation strategies, and collaboration with community services may help to improve the use and satisfaction with this vulnerable population. Other considerations must also be made for patients with behavioral symptoms and cognitive disabilities that may make health care difficult to 
administer in-person and with Telehealth. This may include involving the patient's family when appropriate, improving audio and visual quality, and maximizing the use of community health workers and other facilitators. ${ }^{28}$

\section{CONCLUSIONS}

Telehealth represents a well-researched, efficacious tool to help address this developing crisis. A wide-range of potential interventions have proven effective; however, the success of a Telemental health program relies on numerous internal and external factors. Indeed, the field of digital health interventions may suffer from fragmenting dichotomies, namely randomized versus pragmatic studies, precision versus population health, free market versus regulation, consumer versus clinical uses, big data versus privacy, and open versus proprietary software, which have been proposed by Torous and Haim to have limited the collaboration and expansion. ${ }^{50}$ Having addressed the barriers to implementation, interventions could focus on bringing mental health practitioners into the community via remote Telehealth services, utilizing current community partners to provide the space and technology to perform these visits for a wide range of mental health services, and even scaling up to connect patients with remote mental health practitioners on a region or statewide level. In rural areas that are facing shortages of mental health practitioners and long wait times for patients with mental health disorders, these interventions can be the difference between whether or not patients receive care for their mental illnesses.

Due to the growing deficit of mental health practitioners in many parts of the United States and the ever-growing numbers of individuals struggling with mental illness, new care modalities and interventions must be researched and pursued. This is even more pertinent given the increased mental health burden and barriers to in-person care as a result of COVID-19. As many healthcare professionals and systems make the switch to Telehealth services, it is still imperative to carefully and thoughtfully lay out plans and implement changes for the long term. By researching and better understanding the unique considerations of the patient population and providing appropriate resources, training, and support, a Telemental health program can be highly useful during the pandemic and long after.

Funding statement: This research received no specific grant from any funding agency in the public, commercial, or not-for-profit sectors.

Conflict of interest: The authors declare that there is no conflict of interest.

Contributors: CS performed an extensive search of available journals and articles on the topic, analyzed these findings and then listed, sorted by relevance, categorized, and summarized the literature and key points, statistics, and findings. CS summarized the findings and wrote the initial draft of the manuscript, incorporate and respond to reviewer feedback, and submitted the final version. PP and PN encouraged C S to investigate the role of Telehealth in mental health care and supervised the findings of this work. All authors met frequently to discuss the direction of the research and results and contributed to edits for the final manuscript.

\section{REFERENCES}

1. Mental health by the numbers $\mid$ NAMI: National alliance on mental illness. Nami. org.; 2020 [cited 2020 Jul 18]. Available from: https://www.nami.org/mhstats

2. Follow-up after hospitalization for mental illness-NCQA. NCQA; 2020 [cited 2020 Jul 18]. Available from: https://www. ncqa.org/hedis/measures/follow-up-afterhospitalization-for-mental-illness/ 
3. Perle JG, Nierenberg B. How psychological telehealth can alleviate society's mental health burden: A literature review. J Technol Hum Serv. 2013 Jan;31(1):22-41. https:// doi.org/10.1080/15228835.2012.760332

4. COVID-19 P. Public poll: Emergency care concerns amidst COVID-19. Emergencyphysicians.org.; 2020 [cited $2020 \mathrm{Jul} 18]$. Available from: https://www. emergencyphysicians.org/article/covid19/ public-poll-emergency-care-concernsamidst-covid-19

5. KFF health tracking poll-May 2020 Health and economic impacts. KFF; 2020 [cited $2020 \mathrm{Jul} 18$ ]. Available from: https:// www.kff.org/report-section/kff-healthtracking-poll-may-2020-health-andeconomic-impacts/

6. Talarico I. The use of telehealth to increase mental health services access and promote medication adherence in rural locations. Sigma.nursingrepository.org.; 2020 [cited $2020 \mathrm{Jul} 18]$. Available from: https://sigma. nursingrepository.org/handle/10755/20398

7. Tarlow KR, McCord CE, Du Y, Hammett J, Wills T. Rural mental health service utilization in a Texas telepsychology clinic. J Clin Psychol. 2020 Jun;76(6):1004-14. https://doi.org/10.1002/jclp.22903

8. Mace S, Boccanelli A, Dormond M. The use of telehealth within behavioral health settings: Utilization, opportunities, and challenges. Behavioral Health Workforce Research Center; 2018.

9. Zhou X, Snoswell CL, Harding LE, et al. The role of telehealth in reducing the mental health burden from COVID-19. Telemed e-Health. 2020 Apr 1;26(4):377-9. https:// doi.org/10.1089/tmj.2020.0068

10. Langarizadeh M, Tabatabaei M, Tavakol K, Naghipour M, Moghbeli F. Telemental health care, an effective alternative to conventional mental care: A systematic review. Acta Inform Med. 2017;25(4):240. https://doi. org/10.5455/aim.2017.25.240-246

11. Bischoff RJ, Hollist CS, Smith CW, Flack P. Addressing the mental health needs of the rural underserved: Findings from a multiple case study of a behavioral telehealth project. Contemp Fam Ther. 2004 Jun;26(2):179-98. https://doi.org/10.1023/ B:COFT.0000031242.83259.fa

12. Yuen E, Herbert J, Forman E, et al. Acceptance based behavior therapy for social anxiety disorder through videoconferencing. $J$ Anxiety Disord. 2013;27(4):389-97. https://doi. org/10.1016/j.janxdis.2013.03.002

13. Gros DF, Yoder M, Tuerk PW, Lozano BE, Acierno R. Exposure therapy for PTSD delivered to veterans via telehealth: Predictors of treatment completion and outcome and comparison to treatment delivered in person. Behav Ther. 2011 Jun;42(2):276-83. https://doi.org/10.1016/j. beth.2010.07.005

14. Bouchard S, Paquin B, Payeur R, et al. Delivering cognitive-behavior therapy for panic disorder with agoraphobia in videoconference. Telemed J e-Health. 2004 Mar;10(1):13-25. https://doi. org/10.1089/153056204773644535

15. Jakubowski KP, Jhamb M, Yabes J, et al. Technology-assisted cognitive-behavioral therapy intervention for end-stage renal disease. Transl Behav Med. 2020 Aug 7;10(3):657-63. https://doi.org/10.1093/ tbm/ibz077

16. Stewart RW, Orengo-Aguayo RE, Cohen JA, Mannarino AP, de Arellano MA. A pilot study of trauma-focused cognitive-behavioral therapy delivered via telehealth technology. Child Maltreat. 2017 Nov;22(4):324-33. https://doi. org/10.1177/1077559517725403

17. Carpenter AL, Pincus DB, Furr JM, Comer JS. Working from home: An initial pilot examination of videoconferencing-based cognitive behavioral therapy for anxious youth delivered to the home setting. Behav Ther. 2018 Nov;49(6):917-30. https://doi. org/10.1016/j.beth.2018.01.007

18. Hunkeler EM, Hargreaves WA, Fireman $\mathrm{B}$, et al. A web-delivered care management and patient self-management program for recurrent depression: A randomized trial. 
Psychiatr Serv. 2012 Nov;63(11):1063-71. https://doi.org/10.1176/appi.ps.005332011

19. Tuerk PW, Yoder M, Ruggiero KJ, Gros DF, Acierno R. A pilot study of prolonged exposure therapy for posttraumatic stress disorder delivered via telehealth technology. J Traum Stress. 2010;23(1):116-23. https:// doi.org/10.1002/jts.20494

20. Grubaugh AL, Cain GD, Elhai JD, Patrick SL, Frueh BC. Attitudes toward medical and mental health care delivered via telehealth applications among rural and urban primary care patients. J Nerv Ment Dis. 2008 Feb;196(2):166-70. https://doi. org/10.1097/NMD.0b013e318162aa2d

21. Gibson G, Timlin A, Curran S, Wattis J. The impact of location on satisfaction with dementia services amongst people with dementia and their informal carers: A comparative evaluation of a communitybased and a clinic-based memory service. IPG. 2007 Apr;19(2):267. https://doi. org/10.1017/S1041610206004261

22. Bashshur R, Shannon G, Bashshur N, Yellowlees P. The empirical evidence for telemedicine interventions in mental disorders. Telemed e-Health . 2016;22(2):87-113. https://doi.org/10.1089/ tmj.2015.0206

23. U.S. Substance Abuse Mental Health Services Administration/SAMHSA. Treatment improvement protocol (TIP) 63: Medications for opioid use disorder: Medications for opioid use disorder. Washington, DC: United States Government Printing Office; 2018.

24. Ben-Zeev D, Brian RM, Jonathan G, et al. Mobile health (mHealth) versus clinicbased group intervention for people with serious mental illness: A randomized controlled trial. Psychiatr Serv. 2018 Sep;69(9):978-85. https://doi.org/10.1176/ appi.ps.201800063

25. Zaheer S, Garofalo V, Rodie D, et al. Computer-aided telephone support for primary care patients with common mental health conditions: Randomized controlled trial. JMIR Ment Health.
2018 Dec 10;5(4):e10224. https://doi. org/10.2196/10224

26. HEDIS tips for behavioral health measures. Aetnabetterhealth.com.; 2018 [cited 2020 Jul 18]. Available from: https://www. aetnabetterhealth.com/louisiana/assets/ pdf/providers/HEDIS\%20Tips $\% 20$ for $\% 20$ Behavioral $\% 20$ Health $\% 20$ Measures $\% 20$ H2018.pdf

27. Roberts N, Hu T, Axas N, Repetti L. Child and adolescent emergency and urgent mental health delivery through telepsychiatry: 12-month prospective study. Telemed e-Health. 2017 Oct;23(10):842-6. https://doi.org/10.1089/tmj.2016.0269

28. Dham P, Gupta N, Alexander J, Black W, Rajji T, Skinner E. Community based telepsychiatry service for older adults residing in a rural and remote regionutilization pattern and satisfaction among stakeholders. BMC Psychiatry. 2018 Dec;18(1):316. https://doi.org/10.1186/ s12888-018-1896-3

29. Jacobs JC, Blonigen DM, Kimerling $\mathrm{R}$, et al. Increasing mental health care access, continuity, and efficiency for veterans through telehealth with video tablets. Psychiatr Serv. 2019 Nov 1;70(11):976-82. https://doi.org/10.1176/ appi.ps.201900104

30. Rathbone A, Prescott J. The use of mobile apps and SMS messaging as physical and mental health interventions: Systematic review. FOCUS. 2018;16(4):456-65. https://doi.org/10.1176/appi.focus.16406

31. Fairchild RM, Ferng-Kuo S, Laws S, Rahmouni H, Hardesty D. Telehealth decreases rural emergency department wait times for behavioral health patients in a group of critical access hospitals. Telemed e-Health. 2019 Dec 1;25(12):1154-64. https://doi.org/10.1089/tmj.2018.0227

32. Rui P, Kang K, Ashman JJ. National hospital ambulatory medical care survey: 2016 emergency department summary tables. 2016. Available from: https:// www.cdc.gov/nchs/data/ahcd/nhamcs_ emergency/2016_ed_web_tables.pdf 
33. Telepsychiatry \& telemedicine for behavioral health-Amwell. Amwell; 2020 [cited 2020

Jul 18]. Available from: https://business. amwell.com/service-lines/telepsychiatry/

34. Mohr NM, Vakkalanka JP, Harland KK, et al. Telemedicine use decreases rural emergency department length of stay for transferred North Dakota trauma patients. Telemed e-Health. 2018 Mar;24(3):194-202. https://doi.org/10.1089/tmj.2017.0083

35. Mohr NM, Young T, Harland KK, et al. Emergency department telemedicine shortens rural time-to-provider and emergency department transfer times. Telemed e-Health. 2018 Aug;24(8):582-93. https://doi.org/10.1089/tmj.2017.0262

36. Pangka KR, Chandrasena R, Wijeratne $\mathrm{N}$, Mann M. Exploring the views of emergency department staff on the use of videoconferencing for mental health emergencies in southwestern Ontario. Stud Health Technol Inform. 2015;209:114-20.

37. Takahashi PY, Pecina JL, Upatising B, et al. A randomized controlled trial of telemonitoring in older adults with multiple health issues to prevent hospitalizations and emergency department visits. Arch Intern Med. 2012 May 28;172(10):773-9. https:// doi.org/10.1001/archinternmed.2012.256

38. Meyer JD, McKean AJ, Blegen RN, Demaerschalk BM. Emergency department telepsychiatry service model for a rural regional health system: The first steps. Telemed e-Health. 2019 Jan;25(1):18-24. https://doi.org/10.1089/tmj.2017.0293

39. Roberge J, McWilliams A, Zhao J, et al. Effect of a virtual patient navigation program on behavioral health admissions in the emergency department. JAMA Netw Open. 2020 Jan 29;3(1):e1919954. https://doi. org/10.1001/jamanetworkopen.2019.19954

40. Narasimhan M, Druss BG, Hockenberry JM, et al. Impact of a telepsychiatry program at emergency departments statewide on the quality, utilization, and costs of mental health services. Psychiatr Serv. 2015 Nov;66(11):1167-72. https://doi. org/10.1176/appi.ps.201400122
41. National Consortium of Telehealth Resource Centers. National Consortium of Telehealth Research Centers; 2020 [cited $2020 \mathrm{Jul} 18]$. Available from: https://www. telehealthresourcecenter.org/

42. Komaromy M, Ceballos V, Zurawski A, et al. Extension for community healthcare outcomes (ECHO): A new model for community health worker training and support. J Public Health Pol. 2018;39:203-16. https://doi.org/10.1057/ s41271-017-0114-8

43. Addiction Technology Transfer Center (ATTC) Network. Attcnetwork.org.; 2020 [cited 2020 Jul 18]. Available from: http:// attcnetwork.org/home

44. Ospina-Pinillos L, Davenport T, Mendoza Diaz A, Navarro-Mancilla A, Scott EM, Hickie IB. Using participatory design methodologies to co-design and culturally adapt the Spanish version of the mental health e-clinic: Qualitative study. J Med Internet Res. 2019 Aug 2;21(8):e14127. https://doi.org/10.2196/14127

45. Shore JH. Intended and unintended consequence in the digital age of psychiatry. Psychiatr Clin North Am. 2019 Dec;42(4):659-68. https://doi.org/10.1016/j. psc.2019.08.008

46. Shore JH, Brooks E, Savin DM, Manson SM, Libby AM. An economic evaluation of telehealth data collection with rural populations. Psychiatr Serv. 2007 Jun;58(6):830-5. https://doi.org/10.1176/ ps.2007.58.6.830

47. Shore JH, Schneck CD, Mishkind MC. Telepsychiatry and the coronavirus disease 2019 pandemic — Current and future outcomes of the rapid virtualization of psychiatric care. JAMA Psychiatry. 2020 May 11. https://doi.org/10.1001/ jamapsychiatry.2020.1643

48. Goetter EM, Blackburn AM, Bui E, Laifer LM, Simon N. Veterans' prospective attitudes about mental health treatment using telehealth. J Psychosoc Nurs Ment Health Serv. 2019 Sep 1;57(9):38-43. https://doi. org/10.3928/02793695-20190531-02 
49. Mann DM, Chen J, Chunara R, Testa PA, Nov O. COVID-19 transforms health care through telemedicine: Evidence from the field. J Am Med Inform Assoc. $2020 \mathrm{Jul}$ 1;27(7):1132-5. https://doi.org/10.1093/ jamia/ocaa072

50. Torous J, Haim A. Dichotomies in the development and implementation of digital mental health tools. Psychiatr Serv. 2018 Dec;69(12):1204-6. https://doi.org/10.1176/ appi.ps.201800193
Copyright Ownership: This is an open access article distributed in accordance with the Creative Commons Attribution Non Commercial (CC BY-NC 4.0) license, which permits others to distribute, adapt, enhance this work noncommercially, and license their derivative works on different terms, provided the original work is properly cited and the use is noncommercial. See: http:// creativecommons.org/licenses/by-nc/4.0. 